\title{
Diversity of Olfactory Responses and Skills in Astyanax Mexicanus Cavefish Populations Inhabiting different Caves
}

\author{
Maryline Blin ${ }^{1}$, Julien Fumey ${ }^{2,+}{ }^{1}$, Camille Lejeune ${ }^{1,+}{ }^{\dagger}$, Maxime Policarpo ${ }^{1,3}$, Julien Leclercq ${ }^{1}$, \\ Stéphane Père ${ }^{1}$, Jorge Torres-Paz ${ }^{1}$, Constance Pierre ${ }^{1}$, Boudjema Imarazene ${ }^{1,4}$ and Sylvie Rétaux ${ }^{1, *(1)}$ \\ 1 Paris-Saclay Institute of Neuroscience, CNRS and University Paris-Saclay, 91190 Gif-sur-Yvette, France; \\ blin@inaf.cnrs-gif.fr (M.B.); camille.sabine.lejeune@orange.fr (C.L.); \\ Maxime.Policarpo@egce.cnrs-gif.fr (M.P.); julien.leclercq@u-psud.fr (J.L.); \\ Stephane.pere@inaf.cnrs-gif.fr (S.P.); jorge.torres-paz@cnrs.fr (J.T.-P.); constance.pierre@free.fr (C.P.); \\ boudjema.imarazene@gmail.com (B.I.) \\ 2 Human Genetics and Cognitive Functions, Institut Pasteur, UMR3571 CNRS, Université de Paris, \\ 75015 Paris, France; julien.fumey@pasteur.fr \\ 3 Évolution, Génomes, Comportement et Écologie, IRD, CNRS and Université Paris-Saclay, \\ 91190 Gif-sur-Yvette, France \\ 4 INRAE, Laboratoire de Physiologie et Génomique des Poissons, 35000 Rennes, France \\ * Correspondence: retaux@inaf.cnrs-gif.fr; Tel.: +33(0)-1-69-82-34-52 \\ $\dagger$ Equal contribution to this work.
}

Received: 15 September 2020; Accepted: 8 October 2020; Published: 13 October 2020

\begin{abstract}
Animals in many phyla are adapted to and thrive in the constant darkness of subterranean environments. To do so, cave animals have presumably evolved mechano- and chemosensory compensations to the loss of vision, as is the case for the blind characiform cavefish, Astyanax mexicanus. Here, we systematically assessed the olfactory capacities of cavefish and surface fish of this species in the lab as well as in the wild, in five different caves in northeastern Mexico, using an olfactory setup specially developed to test and record olfactory responses during fieldwork. Overall cavefish showed lower (i.e., better) olfactory detection thresholds than surface fish. However, wild adult cavefish from the Pachón, Sabinos, Tinaja, Chica and Subterráneo caves showed highly variable responses to the three different odorant molecules they were exposed to. Pachón and Subterráneo cavefish showed the highest olfactory capacities, and Chica cavefish showed no response to the odors presented. We discuss these data with regard to the environmental conditions in which these different cavefish populations live. Our experiments in natural settings document the diversity of cave environments inhabited by a single species of cavefish, A. mexicanus, and highlight the complexity of the plastic and genetic mechanisms that underlie cave adaptation.
\end{abstract}

Keywords: fieldwork; wild fish; comparative biology; behavior; troglomorphism; olfactory test; infrared movies; amino acids; chondroitin; plasticity

\section{Introduction}

A very broad diversity of fauna (micro-organisms, insects, vertebrates) lives in underground environments in a more or less permanent manner. Among various niches in the subterranean milieu, caves are emblematic and attractive to human exploration. Species living there permanently display striking phenotypic convergences in their morphology, physiology, or behaviors, with the hallmarks of troglomorphism being the loss of eyes and pigmentation [1]. Caves are often considered as an extreme environment. In the absence of photoautotrophic production, the quantity of food available is limited or 
irregular, the space available is finite, and reproduction seems difficult. Finding food and mates in the absence of vision are the two main challenges faced by cave animals and must limit cave colonization and survival. The evolutionary forces at play during cave adaptation and the respective contributions of natural selection and genetic drift, along with the evolutionary mechanisms, are still a matter of debate [2]. Biologists currently aim at disentangling the roles of genetic mutations and phenotypic plasticity, or epigenetics, in the process. Finally, the observation that some species or lineages have repeatedly adapted to the cave environment while some others never did may support the questioned idea of "pre-adaptive traits" that might favor adaptation to permanent darkness (e.g., [3,4]).

During evolution, most epigean representatives of species that became cave-adapted have become extinct, leaving the underground lineages the only representatives of their taxon, which hampers comparative or genetic studies. The teleost fish Astyanax mexicanus is one of the few exceptions to this rule [5,6]. Therefore, the surface-dwelling and cave-dwelling morphs of this species are increasingly used in evolutionary studies to address the developmental, genetic, or genomic mechanisms of morphological evolution and behavioral adaptation [2,7-9]. The surface form (SF) lives in the rivers of the southern United States and Central America, while the blind and depigmented cave form (CF) is endemic to caves in a karst region located in the states of San Luis Potosi and Tamaulipas in Mexico. There, 30 identified caves host Astyanax mexicanus cavefish populations. They are distributed into three geographically distant groups located, respectively, in the Sierra de El Abra, the Sierra de Guatemala, and the Sierra Colmena $[5,10,11]$. All populations of cavefish and surface fish are interfertile, indicating that they are conspecific $[12,13]$. In nature, the hybridization phenomenon has also been observed and documented [14]. In addition, crosses between geographically distant populations of cavefish can lead to eyed F1 offspring [13], indicating that different mutations are involved in ocular regression in different cave populations and suggesting that some of these populations have evolved independently. However, the evolutionary history of $A$. mexicanus cave populations is still poorly understood because of the geographic dispersion of caves, the lack of knowledge on the underground aquifer network, and the possibility of surface fish introgressions into caves as well as cavefish migrations between caves using underground flows. Recent studies have indicated that initial cave colonization by $A$. mexicanus surface-like ancestors occurred very recently, less than 20,000 years ago $[15,16]$, prompting evolutionary biologists to revise some views about the (rapid) mechanisms of cave adaptation. Like most cave-adapted animals, A. mexicanus cavefish present sensory specializations to life without vision. The brains and sensory systems of surface fish and cavefish differ (reviewed by the authors of [7]), along with their sensory systems: Cavefish have more taste buds [17], more neuromasts [18], and larger olfactory epithelia [14,19,20]. From a behavioral point of view, these mechano- and chemosensory specializations are associated with vibratory attraction behavior to locate moving objects [18] and to an excellent sense of smell to detect low concentrations of food-related odors [20], respectively.

Most of the results described above were obtained in the laboratory, often on one or two lab-raised cave population(s). It is therefore important to extend the studies to other populations, and to validate the results on wild animals in order to avoid possible misinterpretations. Going to the field to observe the natural environment of fish, taking samples, and filming behaviors can help answer questions or revise preconceived ideas [21,22]. For instance, it is often stated that caves are a food-poor environment. However, analysis of stomach contents of wild individuals from the Pachón cave has shown that juveniles feed on small arthropods, and adults on decaying materials and bat guano. Overall and contrary to common belief, Pachón cavefish seemed relatively well fed [23]. Another study revealed that growth curves and age/size relationship are comparable in wild surface fish and wild cavefish, again indicating that cave environment is probably not as food-poor as it may seem [24]. In fact, depending on the location of the cave, its topography, and the hydraulic regime, the amounts of carbon flux can sometimes be of the same order as those reported for surface rivers [1] and, most importantly, the carbon content in the mud sampled from different Astyanax caves can show up to three-fold variation [24]. Energy sources can come from percolating water, animals entering caves and depositing 
their excrement or dead bodies, or rivers that overflow during the rainy season and carry organic matter. These energy sources are both spatially and temporally variable [1]. During several cave expeditions, our team noticed the diversity of local environments between caves-and also between pools within a single cave-reinforcing the idea that field comparisons on fish biology between different caves can be as interesting as the comparison between cavefish and surface fish.

During a field trip in 2013, we carried out, for the first time, experiments of olfactory behavior in situ, in the Subterráneo cave [14]. We had the idea to use a small, light, compact, inflatable children's plastic pool, which was easy to bring on the field. With a rudimentary perfusion system and an infrared camera, we showed that only fish with eyeless phenotype and large olfactory epithelia swam toward an odor source consisting of a food extract. Thanks to this experience and after visits to many other caves, we set up a more complex behavioral experiment, with the aim of systematically assessing olfactory skills and responses of $A$. mexicanus cavefishes inhabiting different Mexican caves. During expeditions in 2016, 2017, and 2019, we performed olfactory tests in the Pachón, Sabinos, Tinaja, Chica, and Subterraneo caves. We found that wild adult cavefish from these five caves showed very variable responses to the three different odorant molecules they were exposed to, with Pachón and Subterraneo cavefish showing the highest olfactory capacities, and Chica cavefish showing no olfactory responses to the odors presented. We discuss these data with regard to the environmental conditions in which these different cavefish populations live.

\section{Materials and Methods}

\subsection{Field Experimentation in Five Different Caves: Constraints and Criteria for Choice}

Caves chosen to carry out olfaction experiments had to fulfill several criteria: (1) Reasonably easy access and climbing challenges, as the total weight of experimental equipment was approximately $25 \mathrm{~kg}$ carried in backpacks and each location had to be visited twice on two consecutive days. (2) Sufficient space inside the cave to install three experimental plastic pools near the water. Thanks to our field experience, we excluded some caves. For example, Curva's ceiling is too low, Chiquitita's entrance is too narrow, as it is located in a big tree's root [10], Toro is a fault in the rock, and Molino and other Guatemala caves are too challenging in terms of climbing ([11] and team observations). (3) Caves already known and visited by the team in the past were preferred to plan the precise place where to install plastic pools and to anticipate troubleshooting. (4) Good representative sampling of diverse local environments (e.g., rocky and muddy caves). (5) Good representative sampling of diverse cavefish population histories (e.g., with or without surface gene flow, or mountain range in which the cave is located). (6) Possibility to compare with our lab studies performed on Pachón cavefish.

Consequently, we decided to perform experiments in four caves: Pachón and Sabinos (fully troglomorphic fish morphotypes in muddy caves), Chica (introgressed fish population in "dirty" cave), and Subterráneo (introgressed population in rocky cave). We also performed some preliminary experiments in Tinaja (fully troglomorphic fish morphotypes in rocky or muddy cave ponds).

\subsection{Cavefishes in the Wild}

Olfactory behavior tests were carried out during three field trips to San Luis Potosi and Tamaulipas States, Mexico, in March 2016, March 2017, and March 2019, in five cave localities. Fieldwork Mexican permits 02438/16, 05389/17 and 1893/19 (to SR and Patricia Ornelas-Garcia) were delivered by the Secretaria de Medio Ambiente y Recursos Naturales. The history of the discovery and precise descriptions of Astyanax caves are given by the authors of [5,11].

The Pachón cave is located in altitude near the village of Praxedis Guerrero $\left(22^{\circ} 37^{\prime} \mathrm{N}\right.$ latitude and $99^{\circ} 01^{\prime}$ W longitude, about $16 \mathrm{~km} \mathrm{SW}$ of Ciudad Mante), in the north Sierra de El Abra, and is easy to access [11]. The cave is small, and the water is stagnant on a muddy bottom. Fish from the Pachón cave (named here CF-Pachón) present a fully troglomorphic type. 
The Sabinos cave is located near the village of El Sabino, in the central Sierra de El Abra $\left(22^{\circ} 06^{\prime} \mathrm{N}\right.$ latitude and $89^{\circ} 56^{\prime} \mathrm{W}$ longitude, about $13 \mathrm{~km}$ NNE of Ciudad Valles) [11]. Villagers installed a padlock grid to exploit this cave and the access is chargeable. The entrance is majestic and the succession of two pits involves bringing harnesses and ropes to abseil down. Fish from the Sabinos cave (CF-Sabinos) are also fully troglomorphic.

The Chica cave is located at the south Sierra de El Abra, on the property of a farmer, at about $21^{\circ} 52^{\prime} \mathrm{N}$ latitude and $89^{\circ} 56^{\prime} \mathrm{W}$ longitude, near the village of El Pujal. It is easy to access, and the entrance and the first cavity are wide. Fish from the Chica cave (CF-Chica) are phenotypically diverse because of surface fish introgression and hybridization. We worked on cavefish from the Chica superficial pool, which are the most troglomorphic/least introgressed among the three natural pools of this cave.

The Subterráneo cave is located in the Micos region, in the Sierra de Colmena $\left(22^{\circ} 03^{\prime} \mathrm{N}\right.$ latitude and $99^{\circ} 14^{\prime} \mathrm{W}$ longitude, about $10 \mathrm{~km} \mathrm{SSW}$ of Micos). Access to this cave is not difficult and the entrance is easy, at the level of the polje or sugar cane field. Fish from the Subterráneo cave (CF-SubT) also occasionally hybridize with surface fish. Hence, they show variable levels of eye regression and pigmentation.

The Tinaja cave is very close to the Sabinos cave, also located in the territory of El Sabino (entrance is free). The cave is located at $22^{\circ} 05^{\prime} \mathrm{N}$ latitude and $89^{\circ} 57^{\prime} \mathrm{W}$ longitude, about $10.5 \mathrm{~km} \mathrm{NE}$ of Ciudad Valles on the Rancho de La Tinaja. Access is via a sugar cane field and permission must be obtained from the owner before crossing it. The cave entrance is accessible after a 2-h hike through a thorny tree forest and a dry canyon covered with jungle. According to Eliott, 2016 [6], the underground hydraulic systems of Tinaja, Sabinos and Sótano de Soyate caves are connected. It has been suggested indeed that the cavefish populations of Tinaja and Sabinos are genetically close $[25,26]$. The cave entrance is majestic. Climbing equipment is not needed but hiking is difficult due to a very slippery mud covering a stony soil. The air is charged with $\mathrm{CO}_{2}$ and renders physical effort somewhat difficult. Fish from the Tinaja cave (CF-Tinaja) show a full cave morphotype.

\subsection{Fishes from the Lab Facility}

Laboratory Astyanax mexicanus surface fish (origin: San Salomon spring, Reeves County, TX, USA) and cavefish (Pachón population) were obtained in 2004 from the Jeffery laboratory at the University of Maryland, College Park, Prince George's County, MD, USA. Here, we also used F1 hybrids, which were the progeny of a cross between a Pachón female and a surface fish male. Colonies were maintained at $22{ }^{\circ} \mathrm{C}$ (cavefish and $\mathrm{F} 1$ hybrids) or $26^{\circ} \mathrm{C}$ (surface fish) on a 12:12 h light:dark cycle. In the present paper, lab-raised fish are named Lab-Pachón, Lab-SF, and Lab-Hyb, respectively. SR's authorization for use of Astyanax mexicanus in research is $91-116$ and the Paris Centre-Sud Ethic Committee protocol authorization number related to this work is $2017-04 \# \sim 8545$. The animal facility of the Institute received authorization 91272105 from the Veterinary Services of Essonne, France, in 2015.

\subsection{Sampling and Photography}

Wild and lab fish were caught with a net (hand net or seine). In order to record their phenotypes after the behavioral tests, wild cavefish were photographed individually in a small aquarium or a plastic support with a graduated ruler and immediately returned to their pond of origin. Total body lengths were measured from these pictures using the ImageJ software. In the Pachón and Sabinos caves, we also weighed the fish using a portable balance. Taring of the balance was carried out with a glass of water, and each fish was weighed inside the glass of water.

\subsection{Odor Choice}

The general principle of the experiment was to place eight fish in an experimental square plastic pool, and to successively perfuse three odorant compounds, each in a different corner of the pool (Figure 1). Each odor perfusion was preceded and followed by a perfusion of water, and a water counterflow flowed permanently from the corner opposite to the perfusion of odor. The odors used 
were L-alanine and L-serine amino acids, and chondroitin (all from Sigma). They correspond to degradation products of organic compounds assimilated to food odors and serve as attractants to fish.

The rationale for choosing alanine and serine as odorant molecules were: (i) To compare them with the results already obtained in the laboratory $[19,20]$ ); (ii) due to the fact that attraction and food-searching behavior are easier to identify than repulsion behavior; (iii) because finding food is a matter of survival for these fish and the sense of smell probably plays a major role in this quest. Indeed, previous experiments performed in the lab had shown that responses to alanine and serine are olfactory-mediated in Astyanax larvae [20].

We chose working concentrations of $10^{-7} \mathrm{M}$ for alanine and serine because it is intermediate between the detection threshold for larvae of surface fish $\left(10^{-5} \mathrm{M}\right)$ and Pachón cavefish $\left(10^{-10} \mathrm{M}\right)$ in the lab. We also reasoned that it would be "risky" to use a very low concentration close to the cavefish detection threshold in noncontrolled cave/field experimental conditions (in particular, water cleanliness, more or less charged with natural odors).

As chondroitin induces freezing in zebrafish [27], we tested this molecule in the lab assuming that A. mexicanus would also adopt a freezing behavior $[28,29]$. Surprisingly, both surface fish and Pachón cavefish instead showed a pronounced and persisting foraging behavior (see Video S1). Preliminary tests in the lab showed that Pachón cavefish have a chondroitin detection threshold at $10^{-4} \mathrm{M}$.

\subsection{Setup Design}

With the conditions for setting up and carrying out the experiments in situ being difficult, the setup was meant to be light, easy to assemble and dismantle, and not too bulky. Plastic pools (Intex, $85 \mathrm{~cm} \times 85 \mathrm{~cm} \times 23 \mathrm{~cm}$; thoroughly rinsed several times in the lab before use to eliminate inorganic volatile odors) were inflated and placed on the ground after adjusting for horizontality with mud or rocks if necessary. They were filled with $40 \mathrm{~L}$ of local cave pond water where cavefish swim. Before pouring into the plastic pools, water was filtered on a coffee filter paper to remove large suspended particles and to ensure cleanliness and good video quality.

A tripod was placed near the corner \#1 of the plastic pool. Two infrared lamps (IR Torch $850 \mathrm{~nm}$, (Maketheone, LA, USA) and the syringe holder were attached to the tripod at a height of $110 \mathrm{~cm}$. The pool was enlightened from above to reduce shadows and filmed from the top to avoid blind spots. For the 2016-2017 campaigns, we built our own infrared camera using a Raspberry pi 3 model B and a Pi-NoIR V2.1 camera. We used a 5-V power bank to supply electricity to this camera, which was controlled from a computer connected in ethernet via a SSH connection. Using Python 2.7.9 and the PiCamera V1.12 package, we wrote two scripts (available at https://github.com/julienfumey/PiCaveRecord) to frame on the pool and to record the video. Videos were converted from h264 to mp4 with VLC software [30]. In 2019, to avoid having to use a computer in caves and to save time, we opted for an infrared hunting camera (nature camera Full HD WK-590, VisorTech, Nairobi, Kenya) which records films in AVI format.

For each plastic pool, we constructed a set of four tubing lines consisting of four 50 -mL syringes connected to a $180-\mathrm{cm}$-long medical solution administration tubing (Infusion device, Intrafix ${ }^{\circledR}$ SafeSet; B. Braun; inside volume of $20 \mathrm{~mL}$ ) and terminated by a $0.6-\mathrm{mm}$-diameter needle (protected by a plastic cap to avoid wounding the fish). The opening of the perfusion was controlled by a Luer stopper and the perfusion rate was regulated by the needle. The end of the tubing was attached to a metal guide (which did not touch the water) to hold the needle in the corner of the plastic pool. Each perfusion line was guided to its respective corner.

A set of three pools was installed in the visited cave, with eight cavefish in each ( $n=24$ total). The installation of three experimental setups was completed in $\sim 4 \mathrm{~h}$ by four people. Fish were left for a 20-h habituation period (from approximately 5 p.m. day 1 to 1 p.m. day 2). The next day, behavioral tests were carried out in the dark (IR recordings) and in silence, in parallel, by three experimenters. 


\subsection{Procedure}

Chemicals were weighed in the laboratory on the day before field trip departure. In a hermetically closed 50-mL tube, $10 \mathrm{mg}$ of L-alanine (CAS 56-41-7, Sigma-Aldrich, Saint-Quentin Fallavier, France), $10 \mathrm{mg}$ of L-serine (CAS 56-45-1, Sigma-Aldrich), and $5 \mathrm{mg}$ or $110 \mathrm{mg}$ of chondroitin (CAS 9082-07-9, Sigma-Aldrich) were each placed (a series of three tubes of powder per cave were thus prepared in advance). Solutions were prepared extemporaneously by adding $50 \mathrm{~mL}$ of filtered cave water to each tube. Chondroitin concentration was $10^{-4} \mathrm{M}$ and $10^{-3} \mathrm{M}$, respectively. For alanine and serine working solutions, a second dilution ( $3 \mu \mathrm{L}$ in $50 \mathrm{~mL}$ ) was prepared to obtain $10^{-7} \mathrm{M}$ solutions. For experiments performed in the lab with surface fish, we prepared a different dilution $(300 \mu \mathrm{L}$ in $50 \mathrm{~mL})$ to obtain $10^{-5} \mathrm{M}$ alanine or serine solutions.

The experimenter seated near the pool took care not to move and not to speak during the whole experiment. To start the experiment, filtered water (=control) was perfused from tubing \#1 and \#3 (i.e., opposite corners), and then from tubing \#2 and \#4 (i.e., opposite corners), for $6 \mathrm{~min}$ each. The aim of this step was to accustom fish to flow and possible vibrations of perfusions and to reduce subsequent nonspecific responses. Indeed, thanks to their lateral line, fish perceive and are attracted by vibrations [18].

Then, water was perfused for $6 \mathrm{~min}$ from tubing \#1 and \#3. Solution flow from the two syringes was initiated simultaneously. When syringes emptied, $50 \mathrm{~mL}$ of alanine solution was added to syringe \#1 and water was added again for counter-flow in syringe \#3 (Figure 1, left panel, orange for alanine). After completion of alanine perfusion, the test continued with a new water perfusion from corners \#1 and \#3. Experimenters took care to ascertain a continuous flow by filling syringes with water or odor solutions before they were completely empty to avoid the introduction of air bubbles into the system.

The same principle was then applied for the perfusion of water, serine or water, and water at corners \#2 and \#4 (Figure 1, middle panel, green for serine). Finally, water, chondroitin or water, and water, were perfused at corners \#3 and \#1 (Figure 1, right panel, blue for chondroitin).

With the exact same setup and procedure, we performed series of experiments in the lab, in a dark and soundproof room, using animals from our breeding facility.

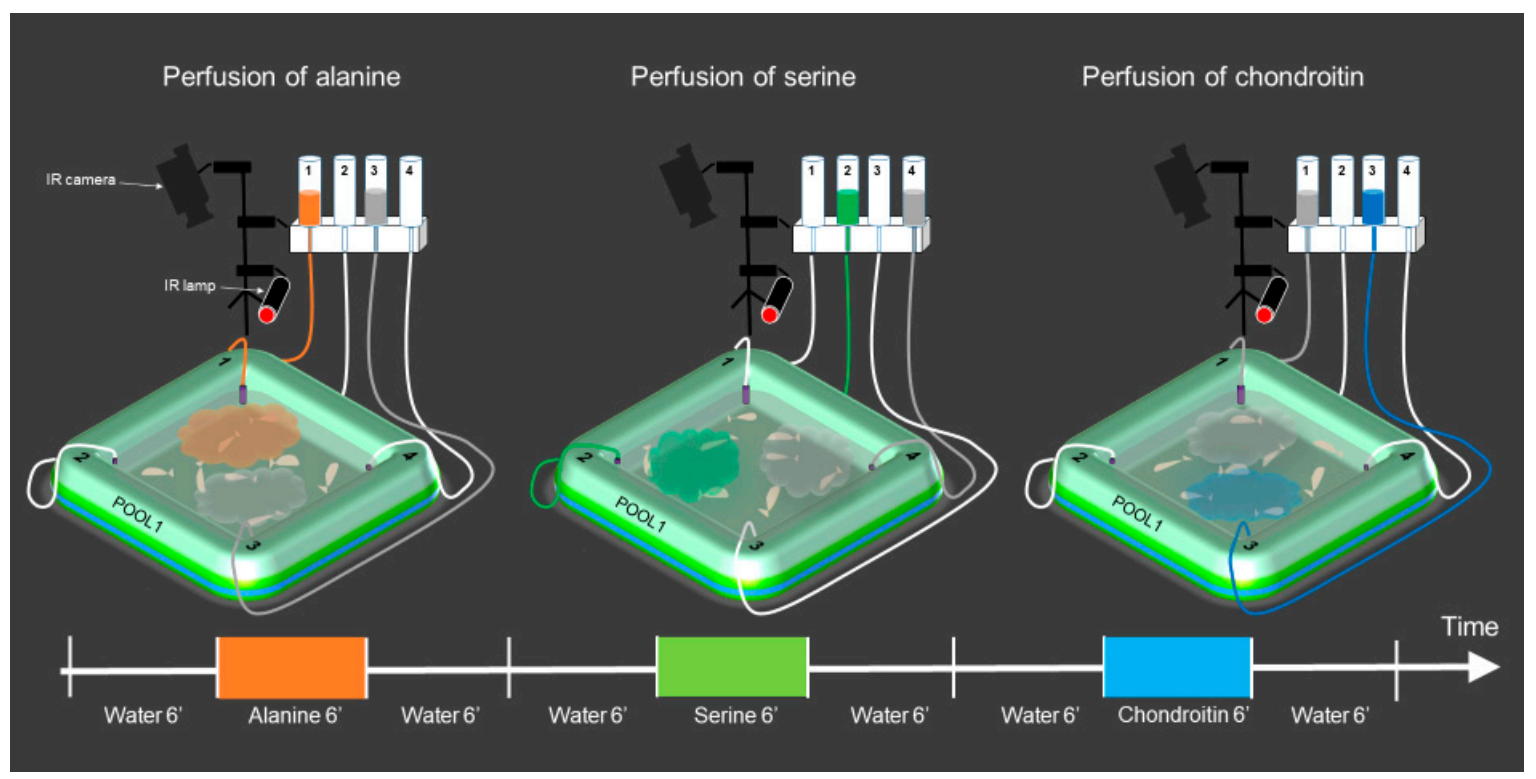

Figure 1. Establishment of a behavioral setup and protocol to test cavefish olfaction in the field. The setup allows testing olfactory responses of eight adult fish in inflatable plastic pools in the dark under infrared recordings. Three different odors were perfused sequentially by gravity flow at different corners of the plastic pool, according to the indicated timeline. See Methods and the first paragraph of Results for details. 


\subsection{Video Scoring}

A total of $\sim 60 \mathrm{~h}$ of infrared videos was recorded. We first attempted to analyze them with an automatic multiple tracking video software [31], which unfortunately did not detect the fish correctly. The reasons were probably numerous: There were eight fish in the pool, the film was in infrared, the contrast of fish on white background was not strong enough, the lamps sometimes produced lighting reflections on the water surface, and the pool edges generated blind spots. Thus, we could not satisfactorily extract the time spent by each fish in each zone and turned to manual scoring.

We first established that, after perfusion of $50 \mathrm{~mL}$ of blue-colored water (with a counterflow), the blue color occupied roughly half of the arena, i.e., a triangle formed by the corner from which the perfusion arrived and the diagonal of the plastic pool (Figure S1A). We therefore considered this half of the pool as the "odorant area" and its opposite half as the "water/control area". Videos were tracked manually through frame-by-frame analysis using Windows Media Player. The number of fish present in each half of the pool was counted every $15 \mathrm{~s}$ during the whole experiment. The odor Preference Index (PI) was calculated for each odor using the formula: (Fish count (odor area) - Fish count (water area)/Total fish count (Figure S1B). When all fish were in the odorant area, PI = 1 (suggesting attraction effect); when all fish were in the opposite corner, PI = -1 (suggesting repulsion effect); and when fish were distributed evenly/randomly in the two parts of the pool, PI = 0 (suggesting no effect).

\subsection{Data Analyses and Statistics}

When the odorous solution was poured into the syringe, $20 \mathrm{~mL}$ (=dead volume contained inside the $180 \mathrm{~cm}$ long tubing) of water flew out before the actual odorant solution entered the pool. For each perfusion, we calculated the speed of the flow to determine odor (or water) perfusion duration. Although tubing were the same length and volume perfused by gravity flow was always $50 \mathrm{~mL}$, the perfusion duration (theoretically $6 \mathrm{~min}$ ) was appreciably variable both between the four perfusion lines, between the successive water/odor/water perfusions, and between the three plastic pools in a given cave. To overcome this problem, we normalized time and calculated PI means over periods corresponding to $25 \%$ of the total perfusion time for each sequence. Thus, we obtained four PI means for each perfusion duration. To determine the statistical significance of the behavioral responses, we used Friedman nonparametric test by ranks for repeated measures in order to compare PI variations along time, during water perfusion before odor, during odor perfusion, and during water perfusion after odor. We also used Wilcoxon Mann-Whitney tests to determine the statistical significance of each PI distribution against PI = 0 (no effect) for each time segment.

Fish sizes and weights were also compared using a nonparametric Wilcoxon-Mann-Whitney test.

Statistical analyses were performed using R 3.6.1 [32] in Rstudio environment [33] with rstatix package, version 0.5.0 [34]. Plots were generated using ggplot2 package [35] from tidyverse open-source R packages [36].

\section{Results}

\subsection{Methodological Considerations: Testing Olfactory Responses in the Lab versus in the Wild}

Our previous analyses of olfactory skills and behaviors in A. mexicanus were mostly performed on larvae, in small 9- $\times$ 13-cm U-shaped "olfaction boxes" containing $150 \mathrm{~mL}$ of water, and under laboratory-controlled conditions and standards $[19,20]$. We had also performed a preliminary experiment on adult fish in the Subterráneo cave but the insights were limited because, among other difficulties, a single "odor" consisting of crushed food pellets was tested as odorant cue [14]. Here, to reach our goal of testing several relevant odors on adult cavefishes inhabiting several caves, we developed a novel setup and a novel experimental procedure adapted to the field.

First, we carried out laboratory experiments to establish and validate the experimental setup (Figure 1 and see Methods) and to study adult fish olfactory behavioral responses. We reasoned that such results would also help us interpreting data obtained in caves. Cavefish from our animal 
facility originated from the Pachón cave but have been raised under markedly different conditions (food, light, water quality) for several generations [37], and they might have been affected by captivity and environment.

Moreover, laboratory experiments were the only option we had to study surface fish olfactory responses. In our experience, it has been impossible to test wild surface fish in the field. Besides the difficulty and time needed for catching wild fish in rivers, they are highly sensitive to manipulations and stress and do not behave "normally," displaying most signs of their stress repertoire [25]. In addition, due to daylight, setting up an experimental test near the river is complex, with a risk of predation on fish by wild animals during the habituation period or destruction/stealing of the equipment.

Finally, we also carried out tests on lab-generated F1 Hybrids. We previously showed that F1 Hybrids larvae have a relatively poor odor detection threshold, similar or even below surface fish skills [19]. In the Subterráneo and Chica caves, wild fish present a wide variety of intermediate hybrid-like phenotypes. It was therefore interesting to perform tests on adult F1 Hybrids in the lab to compare with result obtained on larvae and on wild cavefish populations where hybridization occurs.

\subsection{Responses to Odors in Laboratory-Raised A. mexicanus}

The results of laboratory experiments are presented in Figure $2(n=8$ plastic pools averaged for each graph, hence 64 fish were tested for each morphotype).
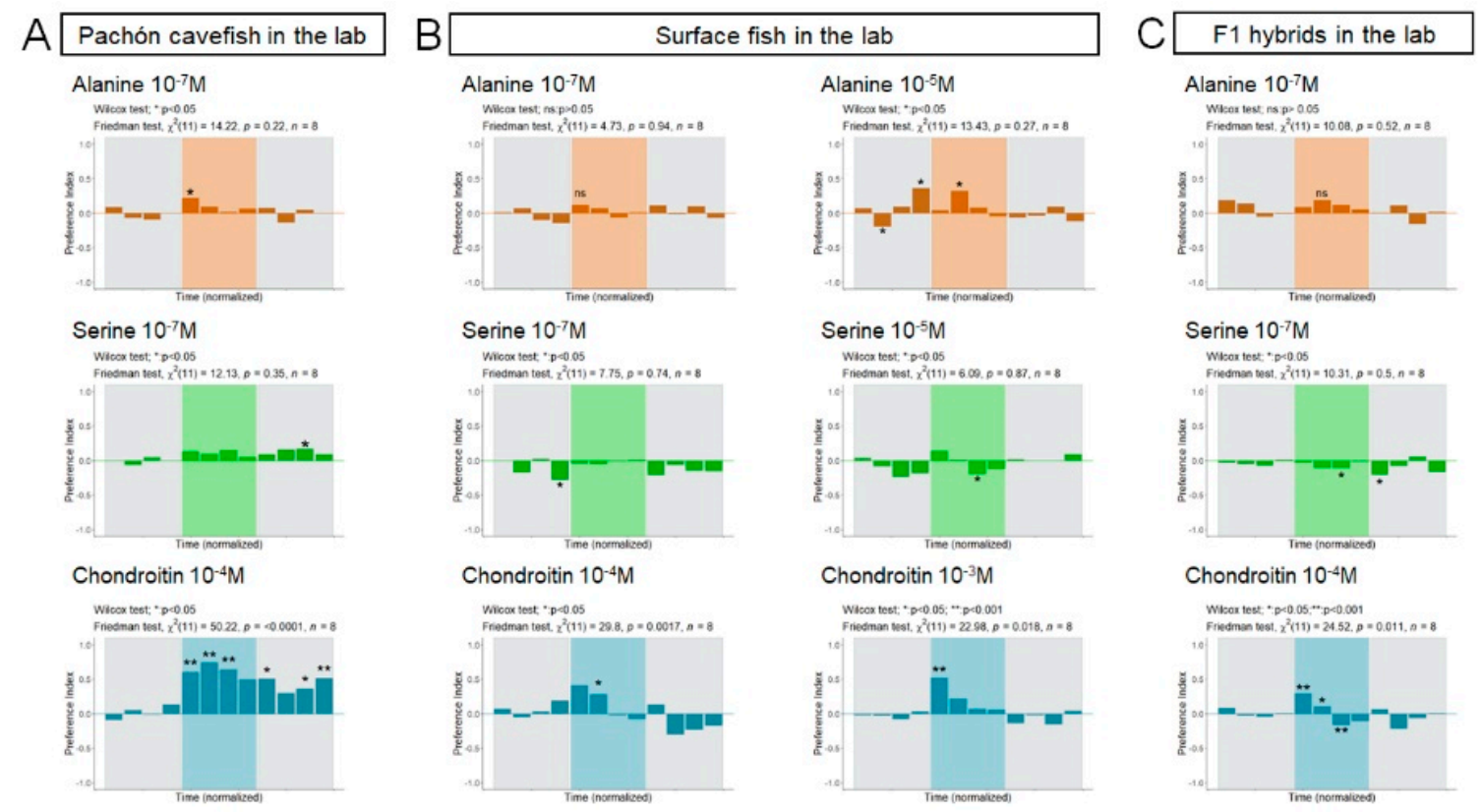

Figure 2. Testing olfactory skills in the laboratory; Olfactory responses of Pachón cavefish (A), Surface fish (B) and F1 hybrids (C) to the indicated odors at the indicated concentrations using the described olfactory setup in laboratory conditions ( $n=8$ for each); The preference index (PI; positive values suggest attraction) is shown as a function of time. Time intervals corresponding to odor perfusion are shaded. Asterisks on graphs indicate significance as compared to no response (i.e., PI = zero) for a given time interval (Wilcoxon Mann-Whitney test). The results of Wilcoxon and Friedman tests to probe the significance of the response across time with repeated measures are also indicated.

In agreement with previous experiments on larvae, only Lab-Pachón showed a positive attractive response to the low concentration of alanine $10^{-7} \mathrm{M}$ (Figure 2A, orange). A modest but significant response was observed during the first quarter of alanine perfusion time (PI $=0.22$; Wilcoxon test, $p=0.008$; but Friedman test, NS), suggesting that Lab-Pachón detected the odor as soon as it arrived in the arena but were not attracted for more than a few minutes. On the other hand, Lab-SF did not 
respond to alanine $10^{-7} \mathrm{M}$ but were significantly attracted by the higher concentration of $10^{-5} \mathrm{M}$ alanine during the second quarter of perfusion time (Figure 2B, orange; PI $=0.32$; Wilcoxon test, $p=0.008$; but Friedman test, NS). These data suggest that, like larvae, adult Lab-Pachón have a better olfactory detection threshold than Lab-SF for alanine. Finally, Lab-Hyb did not respond to the low $\left(10^{-7} \mathrm{M}\right)$ alanine concentration, and were not further tested for a higher concentration (Figure 2C, orange).

Serine elicited very little, if any, response on surface and cave adult Astyanax (Figure 2A-C, green; Wilcoxon tests and Friedman tests, all NS). Of note, Lab-Pachón were present in the odor perfusion zone in a delayed manner, i.e., when water was subsequently perfused from that corner $(\mathrm{PI}=0.16$; Wilcoxon test, $p=0.008$ ), suggesting that the response to serine may have different kinetics compared to the response to alanine. Surprisingly, transiently negative PIs were observed for both Lab-SF $\left(10^{-5} \mathrm{M}\right.$ serine, $\mathrm{PI}=-0.2$; Wilcoxon test, $\left.p=0.008\right)$ and Lab-Hyb $\left(10^{-7} \mathrm{M}\right.$ serine, $\mathrm{PI}=-0.07$ and -0.03 ; Wilcoxon test, $p=0.008$ and 0.02), which was an unexpected result (see Discussion).

Chondroitin provoked strong positive responses in all lab-raised fish, as shown by statistical significance with both Wilcoxon tests and Friedman tests (Figure 2A-C, blue). Lab-Pachón showed intense response to $10^{-4} \mathrm{M}$ chondroitin and were present in the perfusion zone as soon as the odor arrived, with very high PIs ( $\mathrm{PI}=0.6 / 0.7 / 0.6 / 0.5$; Wilcoxon test, $p=0.0004$ at all times). The attraction was persistent, since fish remained in the odor zone during the water perfusion that followed. Lab-SF showed a comparatively more modest response to $10^{-4} \mathrm{M}$ chondroitin (PI $=0.27$; Wilcoxon test, $p=0.008)$, but a strong attraction for the higher chondroitin concentration of $10^{-3} \mathrm{M}(\mathrm{PI}=0.52$; Wilcoxon test, $p=0.0004)$. Finally, the Lab-Hyb were present in the odor zone during the first half of the chondroitin perfusion (PI $=0.29$ and 0.1 ; Wilcoxon test, $p=0.0004$ and 0.008 ) but then seemed to avoid the area (PI $=-0.17$; Wilcoxon test, $p=0.0004$ ). Thus, all morphotypes/genotypes were attracted by chondroitin, but persistence and intensity of the response was particularly spectacular with Lab-Pachón (Video S1). Overall, these laboratory experiments showed that our setup allowed us to measure olfactory responses on adult $A$. mexicanus in a reliable manner.

\subsection{Responses to Odors in Caves, in Wild A. mexicanus Cavefish Populations}

\subsubsection{Pachón Cave}

Despite the thick mud, it was easy to install three plastic pools near the natural main pool (Figure 4B). CF-Pachón were of relatively small size (mean: $4.6 \mathrm{~cm}$ ) compared to other caves studied (Figure 3A, purple) and they were all eyeless and depigmented (Figure 3D). Fish used for olfaction experiments came from the small lateral pool in 2017 and from the main pool in 2019. These two pools communicate with each other when the water level is high and the fish can swim and mix between these two pieces of water. There was no difference in size (or phenotype) between the fish tested during the two expeditions (Figure 3B, purple), suggesting that the local conditions have been stable across the years in the cave. The fauna encountered there and the feeding habits of the CF-Pachón have been described by Espinasa et al., 2017 [23].

Despite fair conditions for installation of the olfactory setup, CF-Pachón from two experimental pools out of the six recorded presented an obvious place preference behavior (one in 2017 and one in 2019), for unknown reasons (Figure 5). These data were thus discarded from the analyses shown in Figure 6A. Contrary to Lab-Pachón, CF-Pachón did not respond to alanine $10^{-7} \mathrm{M}$. Even more so, the preference index reached negative values during the perfusion. Conversely, CF-Pachón were strongly and significantly attracted by serine $10^{-7} \mathrm{M}(\mathrm{PI}=0.41)$ and by chondroitin $10^{-4} \mathrm{M}(\mathrm{PI}=0.55)$, as shown both by Friedman and Wilcoxon statistical tests (Figure 6A, green and blue). Moreover, the type of behavioral response elicited by chondroitin in the field was similar to the food-seeking behavior recorded in the lab (Video S2). Although slightly surprising because the alanine and serine responses seem divergent between the Lab-Pachón and the CF-Pachón, these data confirm, in the field, that CF-Pachón have excellent olfactory detection skills. 

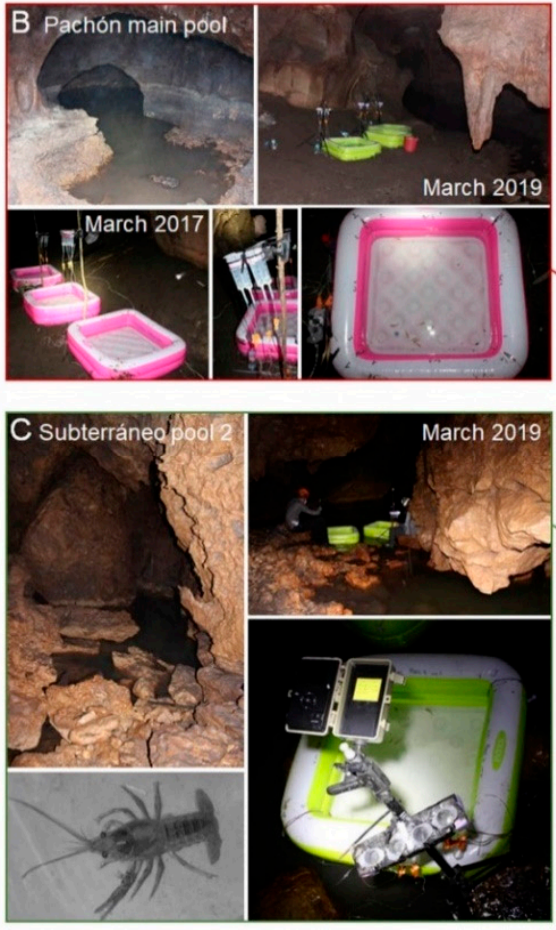

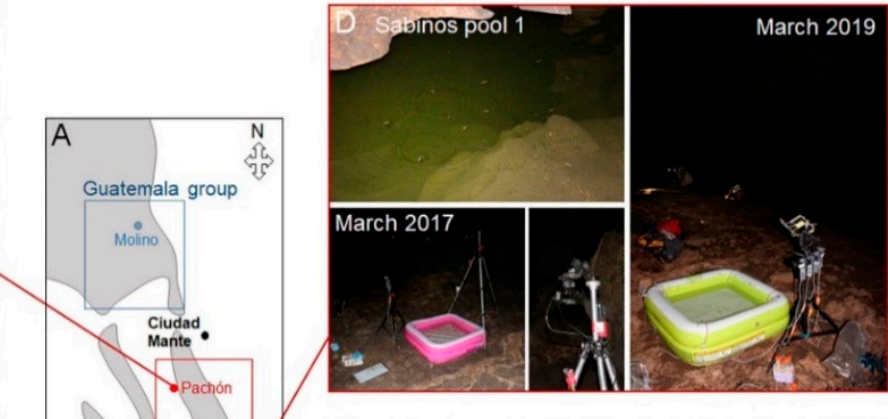

政

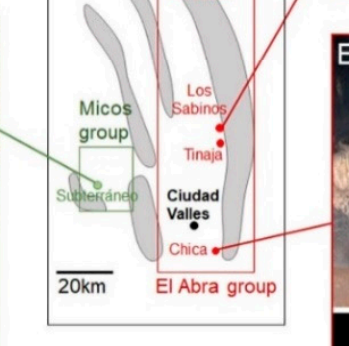

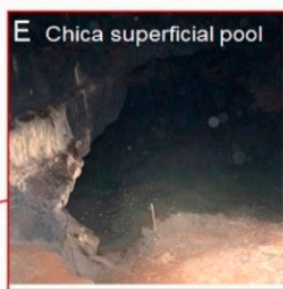
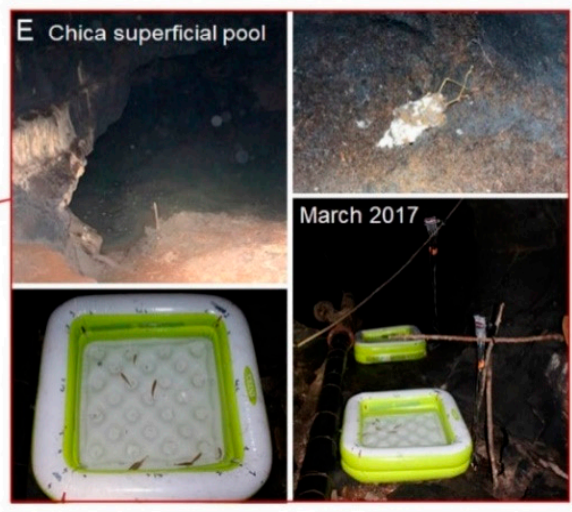

Figure 3. Cavefish sizes and phenotypes in different caves; (A) Sizes (left graph) and body mass indexes (BMI, right graph) of the cavefish individuals tested for olfactory responses in the Pachón (purple), Sabinos (brown), Subterráneo (red), and Chica (grey) caves. Asterisks indicate significant differences (Mann-Whitney test); (B) Comparison of sizes of the cavefish tested for olfactory responses in different field campaigns, in 2017 and 2019, showing that the condition of the fish tested did not vary; (C) Comparison of sizes of cavefish in different water ponds in a same cave locality, showing that fish condition varied, probably due to local trophic, environmental and/or genetic parameters; (D) Fish phenotypes are fully troglomorphic in the Pachón and Sabinos caves, whereas hybrid-type fish with small eyes (arrowheads) and some pigmentation can be seen in Subterraneo (pool 2) and Chica (superficial pool) caves. They result from hybridization with introgressed surface fish. Of note, in Subterraneo, surface fish enter by the cave entrance at the polje level, whereas, in Chica, surface fish enter the cave by the bottom. Hence, in both caves, the most troglomorphic fish are larger. 


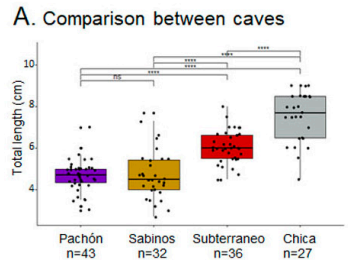

B. Comparison between years

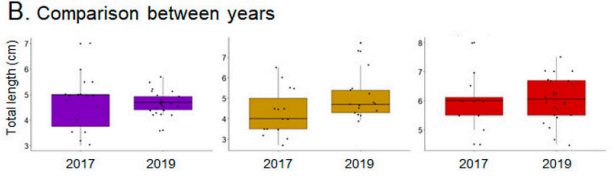

C. Comparison between pools within a cave

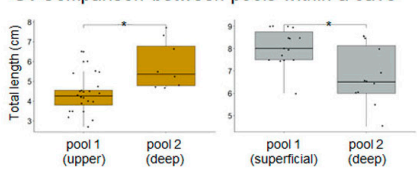

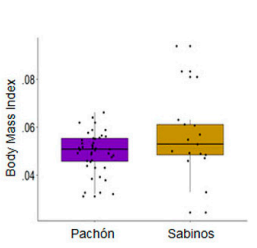

D. Fish phenotypes
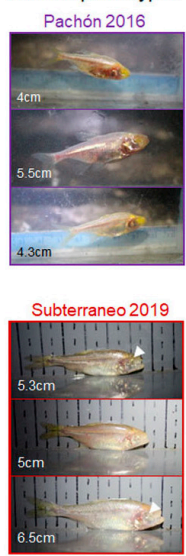

$\underline{1 \mathrm{~cm}}$
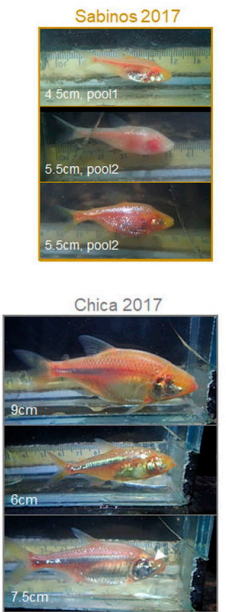

Figure 4. Sampling cavefish olfactory skills in their natural environment; (A) Simplified map of the region of Ciudad Valles, Mexico. Mountain ranges are in grey. The locations of visited caves are indicated by colored circles. The color code indicates the geographical group where they belong; (B) Fieldwork in the Pachón cave. The main pool was muddy and the water level was low. Note that in March 2017 and March 2019, the three plastic pools were installed and processed in the best possible reproductive manner, on the "beach" along the main pool. Close-ups show the Luer-lock perfusion system and the eight fish installed in one of the experimental plastic pools; (C) Work in pool 2 of the Subterráneo cave, located just after the 3-m pit. This pool offers a rocky substrate. There, we repeatedly observed the presence of crayfishes (predators on adult cavefish). The bottom right panel shows the infrared, hunting-type camera, used in our 2019 campaign; (D) Work in Los Sabinos cave. Pool 1 of this cave had crystal-clear water. Again, note that olfactory setups were installed in the same place in March 2017 and 2019, respectively, i.e., on a rocky plateau just above pool 1. A close-up shows the Raspberry Pi camera used during our 2017 campaign. (E) Testing olfaction on fish from the Chica superficial pool. The top photos show the guano slope leading to the superficial pool, which was very rich in organic material (decomposing bat cadaver). On the bottom left panel, note the large size of the eight fish (compare with the equivalent picture in Pachón, and see Figure 3).

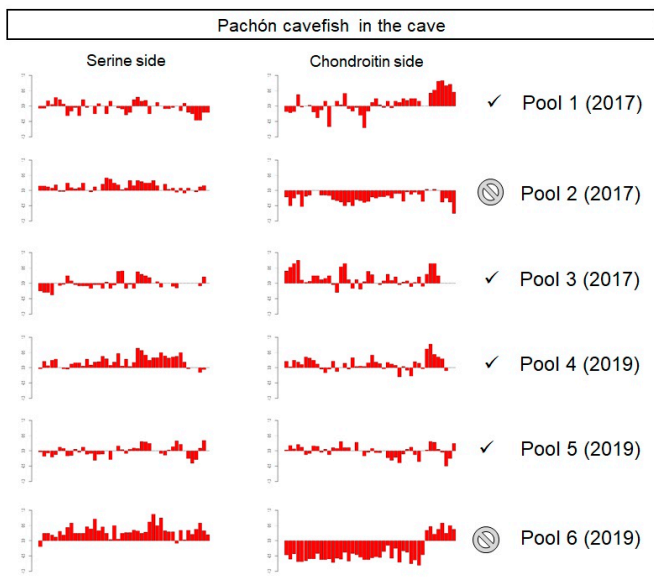

Figure 5. The place preference problem during cave experimentation. Graphs showing the position of the fish along the whole $\sim 1 \mathrm{~h}$ protocol for the $\mathrm{n}=6$ experiments performed in the Pachón cave. The bars represent the preference of fish toward the serine perfusion side (left column) or the chondroitin perfusion side (right column). In theory, the eight fish should swim across the arena and distribute or explore randomly except for the response to the considered odor. However, in some instances (here, plastic pools 2 and 6), it was not the case: The fish remained in the same part of the plastic pool throughout the experiment, showing place preference for unknown reasons. These data were excluded from analysis. 


\subsubsection{Sabinos Cave}

The first fish pool encountered, i.e., the upper pool, lies under a large arch and contains shallow water that disappears under the rock (Figure 4D). Exploration there revealed a large (20-30 m), continuous body of water under a low ceiling, hosting a significant cavefish population. The water was clear with a muddy substrate. Juvenile cavefish (size 1-2 cm) were observed, indicating that reproduction occurs. Large isopods and numerous mysid shrimps were present. Just above this natural pool, a natural rocky stage allowed us to install three plastic setups in the same place in 2017 and 2019 (Figure 4D). From there, a corridor led into a large and relatively low room where a second fish pool, or bottom pool, was encountered. Bats were numerous (observed in 2013, 2017, and 2019). The air was loaded with spores and the soil was covered with patches of microorganism covered with insects. $\mathrm{CO}_{2}$ was not measured but was probably high.

All fish in the Sabinos cave had a fully troglomorphic phenotype (Figure 3D). Fish used in the experiments were sampled from both the upper and the bottom pools. Like in the Pachón cave, their size and body mass index was modest (Figure 3A, brown; mean: $4.7 \mathrm{~cm}$ ) and did not vary between 2017 and 2019 samplings (Figure 3B, brown), suggesting that the two experimental series were performed on fish of similar condition. We noted that CF-Sabinos found in the deeper pool were significantly larger (Figure 3C, brown) and more corpulent (Body Mass Index, Wilcoxon test, $p=0.006$ ) than those fished in the upper pool, suggesting that they were older [24] and/or better fed. However, this observed difference in condition could not be correlated to their olfactory responses. As in the Pachón cave, we observed a strong place preference bias in two experiments out of six, hence these data were discarded (Figure S2). When tested for olfactory responses, CF-Sabinos were attracted neither by alanine nor by serine (Figure 6B). However, they did spend time in the odorous part of the arena after chondroitin perfusion (PI values between 0.46 and 0.28 ; Wilcoxon test, $p=0.02$; but Friedman test, NS), suggesting that they were, although moderately, attracted to this molecule (Figure 6B).

\subsubsection{Subterráneo Cave}

A 20 min descent in a boulder tunnel filled with organic waste (including of very large size like trees) carted inside during the rainy season leads to a first small fish pool, where almost all individuals are surface-like (A. mexicanus, poecilids, some cichlids, presumably washed in during flooding) and where troglomorphic fish are rare (see Simon et al., 2017 [24]). Then, the tunnel continues and leads to a small 5-m pit which descends directly into the natural pool 2, with clear water on a rocky substrate, where CF-SubT swim. All fish used and measured in 2013 [14], 2016, and 2019 came from this room (Figure 4C). The ground of the cave at this level was relatively horizontal but it was covered with pebbles. As there was no mud to flatten the ground, we installed plastic pools on small flat mounds (Figure 4C).

As we previously described [14], CF-SubT presented mixed phenotypes in terms of eye size and pigmentation as a result of hybridization with introgressed surface fish, and most of them were not fully troglomorphic (Figure 3D). We found that CF-SubT were significantly larger than CF-Pachón and CF-Sabinos (Figure 3A, red; mean: $6 \mathrm{~cm}$ ), suggesting that they may have been overall slightly older [24] and/or in better nutritional condition. In addition, the CF-SubT tested in 2017 and 2019 were of similar sizes (Figure 3B, red). During the olfaction tests, the CF-SubT were present in the $10^{-7} \mathrm{M}$ alanine perfusion area approximately $2 \mathrm{~min}$ after the odor arrived and they remained in this zone during the entire perfusion time (PI $=0.25 / 0.26 / 0.19$; Wilcoxon test, $p=0.007 / 0.118 / 0.025$ ) (Figure 6D). Conversely, they did not significantly respond to serine. Finally, CF-SubT were attracted by chondroitin (but note that the concentration perfused was higher than in other caves: $10^{-3} \mathrm{M}$ ), since they stayed in the odorous area with high preference indexes (PI between 0.48 and 0.33; Wilcoxon test, $p=0.025 / 0.007$ ). Thus, overall, CF-SubT showed significant olfactory skills and responses in their natural settings. Of note, in videos, it was impossible to make any correlation between olfactory responses and the degree of troglomorphism exhibited by individual CF-SubT. 

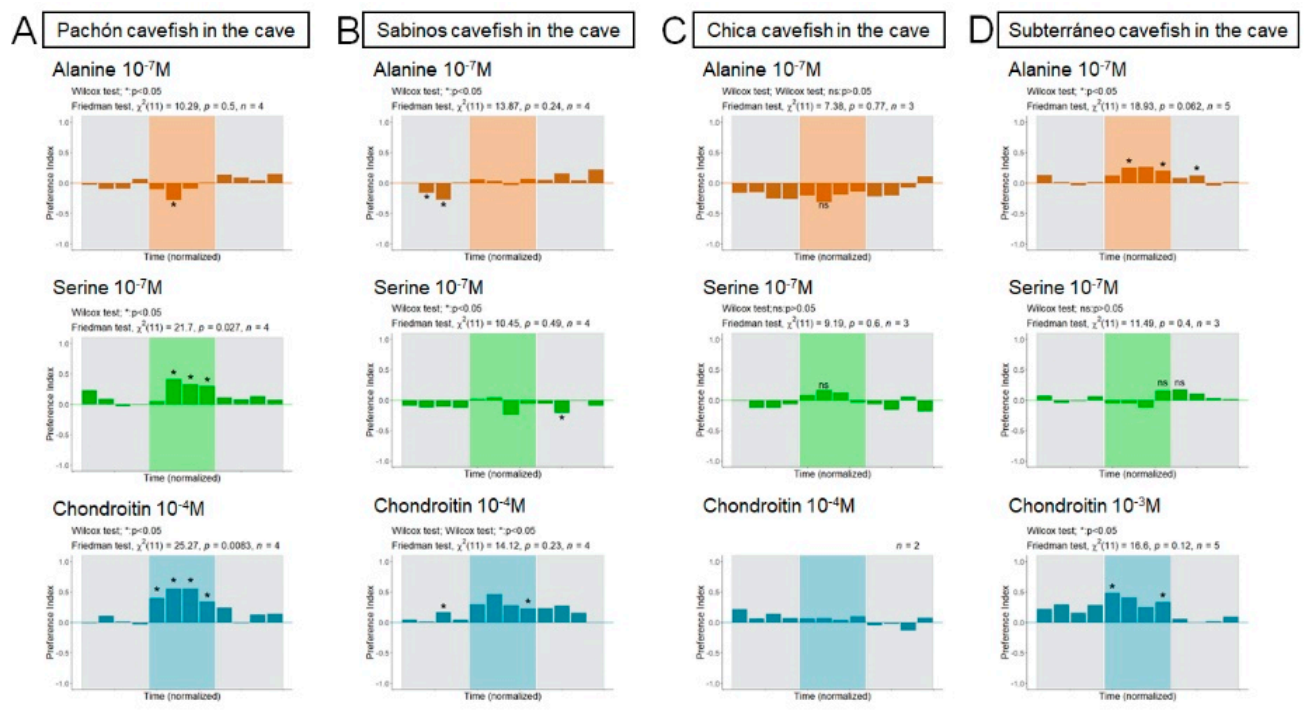

Figure 6. Olfactory responses of cavefishes in natural cave settings; (A-D) Olfactory responses recorded in the Pachón (A), Sabinos (B), Chica (C), and Subterráneo (D) caves; Odors and concentrations are indicated, as well the number of replicates. The preference index (PI; positive values suggest attraction) is shown as a function of time. Time intervals corresponding to odor perfusion are shaded. Asterisks on graphs indicate significance as compared to no response (i.e., PI = zero) for a given time interval (Mann-Whitney test). The results of Wilcoxon and Friedman tests to probe the significance of the response across time with repeated measures are also indicated.

\subsubsection{Chica Cave}

The Chica superficial pool (or pool 1) hosting cavefishes is a large body of water with a guano slope bank, probably very rich in organic content due to a large bat colony and influx of organic materials from the surface during the rainy season (decaying bats, vegetal debris were observed; Figure 4E). A corridor with a flat rocky floor allowed us to set up experiments just above this superficial pool (Figure 4E). Further down, Chica pool 2 could be reached by following the underground river, which cascaded after a pit [11], and also contained fish that were phenotypically less troglomorphic and more surface-like or hybrid-like than the fish in superficial pool 1 (not shown).

The superficial pool CF-Chica tested were not fully troglomorphic, i.e., some individuals showed some degree of pigmentation and had tiny to small eyes (Figure 3D), probably as a result of hybridization with surface fish. CF-Chica were, by far, the largest and most corpulent fish that we tested among the different caves visited (Figure 3A, grey and Figure 3D). The individuals fished for the olfaction experiments originated from the superficial pool, where the most extreme sizes (mean: $8.1 \mathrm{~cm}$, max: $9 \mathrm{~cm}$ ) were encountered (compare with CF-Chica from pool 2; mean: $6.8 \mathrm{~cm}$, Figure 3C, grey). In this cave, olfaction experiments were performed once, in 2017. Hence, only $n=3$ plastic pools were recorded. For the CF-Chica, Friedman tests indicated that the Preference Indexes did not vary between the perfusions of water and amino acids, and the Wilcoxon tests indicated that they were not different from 0 (Figure $6 \mathrm{C}$ ). A video problem at the end of one recording resulted in $n=2$ for chondroitin, for which we could not perform statistical tests. However, for chondroitin, the pattern was flat along the whole perfusion sequence, and there was no such behavioral foraging response as visually observed in other caves. These results showed that CF-Chica did not respond to the odors used in our tests, including chondroitin that elicited strong responses in all other cavefishes tested as well as in Lab-SF and Lab-Hyb.

\subsubsection{Tinaja Cave}

The first, very small, Tinaja "perched pool" was encountered after a 20-min walk and consisted of a small piece of crystal-clear water retained between rocks (Figure 7A). We found fully troglomorphic 
CF-Tinaja there (approximately $n=20-30$ ) in two consecutive years (2016 and 2017). After another 20 min of hiking down, the tunnel narrows before reaching a fault, at the bottom of which lies Traverse Lake (or Tinaja pool 1) (Figure 7B). A small natural beach allowed for experimental installation, but the space was small and inconvenient ( $\sim 3 \mathrm{~m}$ wide). Drops of water constantly falling from the ceiling generated a very thick and sticky layer of mud. The water was cloudy. In such conditions, it was difficult to install the olfaction setup. This was partly achieved in March 2016, with only $n=2$ plastic pools and with video recordings performed in the light (Figure 7B). The size of the fish was $6.1 \mathrm{~cm}$ on average ( $n=12$ measured). In 2017, we aimed to complete the Tinaja study and we performed $n=1$ additional olfactory test directly in the small natural "perched pool 1." Although we cannot provide statistical support ( $n=2$ and $n=1$, respectively), the video analyses suggest that that CF-Tinaja were not attracted by any of the three perfused odors (data not shown).
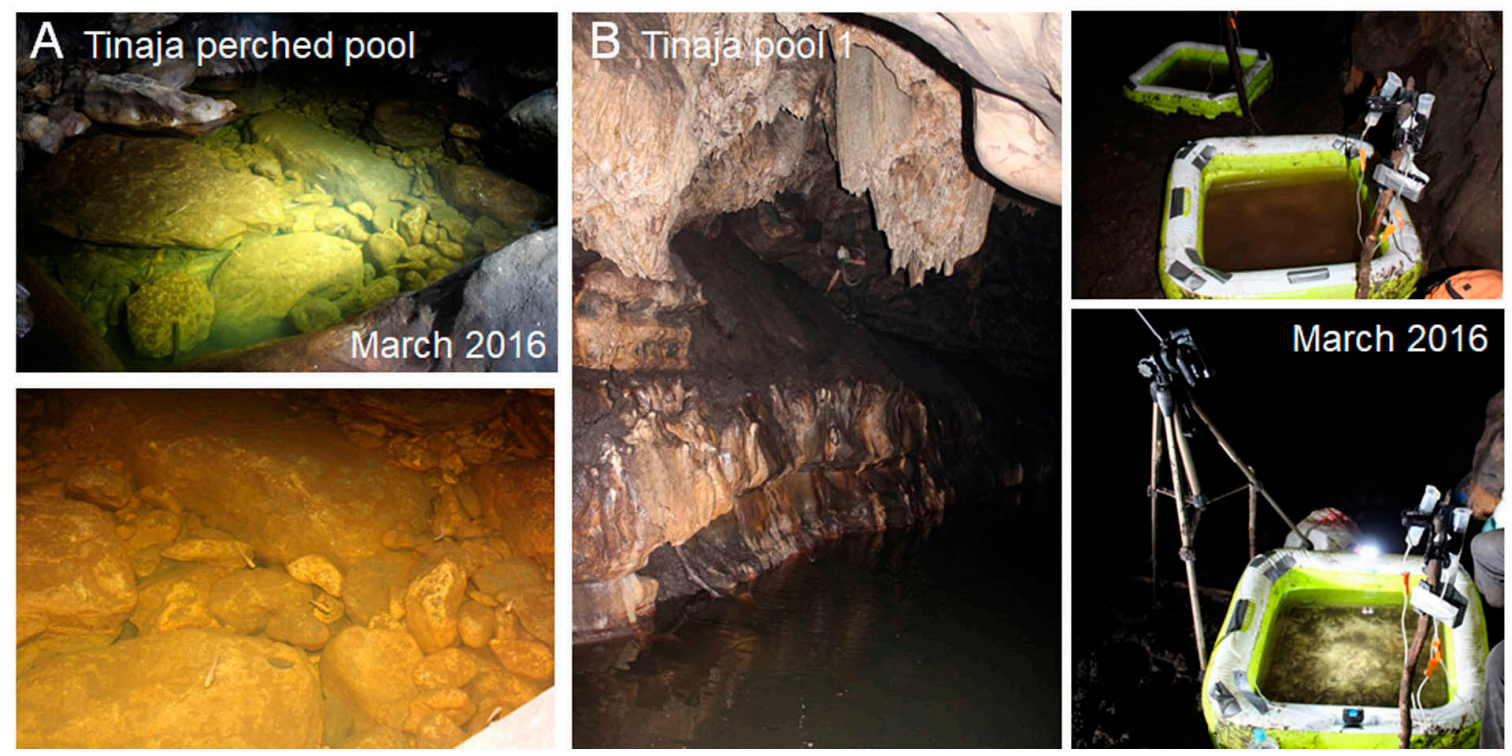

Figure 7. Experimentation in the Tinaja cave; (A) single test was "manually" performed in Tinaja perched pool, a very small $\left(\sim 3-4 \mathrm{~m}^{2}\right)$ and shallow natural pool where water is retained between rocks. There, over the years, we repeatedly observed 20-30 fully troglomorphic fish, presumably trapped there during the rainy season. The water is crystal clear; (B) Preliminary experiments were performed in March 2016 in Tinaja pool 1 (called Traverse Lake), a relatively large and muddy water reservoir. At the time, films were recorded in the light (instead of in the dark with infrared) and the perfusion system and the odorant molecules perfused were still under tuning. This type of expedition was of paramount importance to improve and establish the final experimental setup and design we used successfully in 2017 and 2019 in other caves.

\section{Discussion}

\subsection{Testing Cavefish Olfaction in the Field: A Challenge}

To our knowledge, this is the first study reporting systematic, precise, and quantified olfactory responses to specific odorant molecules in behavioral tests with subterranean animals in their natural environment (but see $[14,22])$. The trip organization, transport of equipment, and difficulties of access, together with the struggle of setting up experiments in "natural laboratories" which were different each time and handling the unexpected, means that few researchers risk of this kind of experimentation. The interpretation of the results must also be approached with caution because the experimental parameters are far from being fully mastered or even known. Of note, we found that establishing and pretesting of protocols in the lab are mandatory steps, and the comparison between lab and field results can help reach conclusions. Finally, this type of field behavior work needs to extend 
over the long term, with continuous improvements and feedbacks. For example, the difficulties we encountered in Tinaja in 2016 allowed us to improve the setup. From that expedition, we concluded that the plastic pools needed to be perfectly clean and the cave water needed to be filtered before being poured into the arena or else the videos would be difficult to interpret, that we should not use rechargeable batteries for infrared lamps because the Mexican electrical network sometimes fluctuates, that we should systematically include a water counterflow at the corner opposite to odor perfusion to remove biases of lateral line driven behaviors, and that we should not plan for 2-day-long experiments in a cave where access is difficult. Hence, our Tinaja 2016 experiments ( $n=2$ only) were not performed in the dark, did not have the same perfusion sequence nor the same odor concentrations, and were not included in the main results of the present paper, but they ended up being very instructive for improving the setup and protocols, and they allowed us to draw preliminary conclusions (see Figure 7).

\subsection{Alanine, Serine and Chondroitin Elicit Variable Behavioral Responses in Wild and Lab-Raised Adult A. mexicanus}

The choice of odors presented to fish was critical. Whereas alanine and serine amino acids are "classically" used in fish olfaction experiments because they correspond to food degradation products and therefore serve as food-related attractive cues (e.g., [38-40]), the use of chondroitin is more novel.

Chondroitin sulfate is a glycosaminoglycan composed of a chain of alternating sugars and a component of the cartilage matrix. It is also present in zebrafish skin mucus, where it was recently discovered to serve for the long-searched active molecule of the alarm substance [27,41]. Indeed, zebrafish strongly react to chondroitin with a typical alarm behavior, including erratic swimming and freezing, and the activated brain regions are the same as those activated after presentation of skin extracts [27]. In A. mexicanus, it was therefore unexpected that chondroitin would elicit (1) attraction and (2) foraging, and even more so, (3) the same response in the two morphs. Along with the idea that the alarm substance is species-specific [42], our findings suggest that its composition in zebrafish and Astyanax must be markedly different. Moreover, early studies have suggested that alarm reaction is indeed present in surface fish but mostly lost in cavefishes, at least those originating from the Pachón and Chica caves $[28,29,43]$. Here, surface fish and Pachón cavefish strongly reacted to chondroitin, but Chica cavefish did not, further suggesting that this molecule is not part of the alarm substance in the species. Rather, it seems to correspond to a foraging cue, which is in line with Astyanax being carnivorous, eating carcasses and responding to cartilage odors. In fact, we ended up considering chondroitin as a sort of positive control: All types of fish tested, in the lab and in the field (except CF-Chica, see below), adopted an intense food search behavior, almost leaking or sucking the extremity of the tube where chondroitin flew in (Supplementary videos). Of note, chondroitin was the third and last odor presented in the protocol because we reasoned that the more modest responses elicited by amino acids may be lost after the strong stimulation caused by chondroitin. Finally, response to chondroitin also validated, in a way, the whole test, and we were confident that absence of response to amino acids sometimes observed was true because fish reliably and repeatedly responded to chondroitin at the end of the behavioral assay.

Regarding amino acids, the results we obtained with adult fish in the lab globally corroborate what has already been observed with 1-month-old larvae [20]: The adult Lab-SF had a lower olfactory detection threshold than the Lab-Pachón (Table 1). The results confirm that older and larger adult fish do not have a better detection threshold than 1 month-old-larvae [19], reinforcing the idea that olfactory detection threshold is not directly or exclusively linked to the size of the olfactory organs, or to the intrinsic properties of the olfactory system. The results also confirm that the olfactory system of the 1-month-old larvae tested in our previous studies was functionally mature.

For reasons explained earlier, adult surface fish (and F1 hybrids) could only be tested in the lab. We considered their olfactory performance to be poor (Table 1). They did respond to chondroitin-albeit less intensely than Lab-Pachón, but barely responded to amino acids, even at high concentrations. An explanation to this mitigated result might be that the olfactory response is slightly blurred by 
schooling behavior, even in the dark $[25,44]$. Indeed, for these fish, we observed transient statistically significant PI scores (positive or negative) before or after odor perfusion (see Figure 2B,C) that cannot correspond to true and specific olfactory responses, and that probably resulted from the grouping of all eight fish in the same area of the arena at some time points of some experiments. These observations further confirm the appropriateness of using 1-month-old surface fish-which do not yet school intensely-for olfactory tests with these morphotypes. Of note, such an interference of schooling in olfactory responses is irrelevant in the case of cavefish who have lost schooling behavior, as first observed by Parzefall in the field [22].

Table 1. A summary of the results obtained in all the cave and laboratory olfaction experiments. Attractive response (+) or no response (0) is summarized for each cavefish or surface fish population, when recorded in the field or in the lab (as indicated in the different columns) for the 3 different odors studied (as indicated in the different rows; orange/alanine, green/serine, blue/chondroitin). Light and dark colors indicate response to the high concentration (light color, lower detection capacities) or the low concentration (dark color, better detection capacities) of the odor considered. The last line (grey shades) indicates to how many odors, out of the 3 tested, the fish have responded.

\begin{tabular}{|c|c|c|c|c|c|c|c|c|}
\hline \multirow{2}{*}{$\begin{array}{l}\text { Attraction : }(+) \\
\text { No response : }(0)\end{array}$} & \multicolumn{5}{|c|}{ Cave fishes } & \multicolumn{3}{|c|}{ Surface fishes } \\
\hline & $\begin{array}{c}\text { Lab- } \\
\text { Pachón }\end{array}$ & $\begin{array}{c}\text { CF- } \\
\text { Pachón }\end{array}$ & $\begin{array}{c}\text { CF- } \\
\text { SubT }\end{array}$ & $\begin{array}{c}\text { CF- } \\
\text { Sabinos }\end{array}$ & $\begin{array}{l}\text { CF- } \\
\text { Chica }\end{array}$ & $\begin{array}{l}\text { Lab- } \\
\text { SF }\end{array}$ & $\begin{array}{l}\text { Lab- } \\
\text { SF }\end{array}$ & $\begin{array}{l}\text { Lab- } \\
\text { Hyb }\end{array}$ \\
\hline Alanine & & & & & & & & \\
\hline $10^{-7} \mathrm{M}$ & + & 0 & + & 0 & 0 & + & 0 & 0 \\
\hline Serine & & & & & & & & \\
\hline $10^{-7} \mathrm{M}$ & + & ++ & 0 & 0 & 0 & 0 & 0 & 0 \\
\hline Chondroitin & & & & & & & & \\
\hline $10^{-4} \mathrm{M}$ & +++ & +++ & ++ & + & 0 & ++ & + & + \\
\hline $\begin{array}{l}\text { Olfactory performance } \\
\text { summary }\end{array}$ & $3 / 3$ & $2 / 3$ & $2 / 3$ & $1 / 3$ & $0 / 3$ & $2 / 3$ & $1 / 3$ & $1 / 3$ \\
\hline
\end{tabular}

Finally, the comparative responses of Pachón cavefish from the lab and from the field to amino acids is also worth discussing. Lab-Pachón responded positively (=attraction, like larvae) to alanine, whereas CF-Pachón responded negatively (=repulsion). Moreover, Lab-Pachón response to serine was weak or delayed (if any), whereas CF-Pachón response was strong and immediate. Thus, both lab-raised and wild animals originating from the Pachón cave seem able to detect very low concentrations of amino acids (here, $10^{-7} \mathrm{M}$ ), thereby confirming their excellent olfactory skills. However, unexpectedly, their responses to a given odor can differ in nature or intensity. The first hypothesis we can draw relates to the distinct environment in which the lab and wild fish were grown and live, e.g., the water parameters or diet could be at the origin of their difference in reaction. Indeed, the water used in the lab tests (tap water bubbled for $24 \mathrm{~h}$ ) is "cleaner" than the water used in the field, which, although filtered, is loaded with dissolved organic compounds. It has also been proposed that fish move toward or away from a given amino acid depending on the concentration as well as the age and the species of fish. For example, cysteine acts as a repellant for 1-month-old juvenile zebrafish $\left(3 \times 10^{-5} \mathrm{M}\right.$; [45]) but as an attractant for rainbow trout $\left(10^{-6} \mathrm{M}\right.$; [39]). Moreover, olfactory conditioning or learning seems to play an important role in the expression of behavioral responses $[46,47]$. In the Pachón cave, juveniles feed on micro-arthropods and adults feed on decomposing organic debris and mud [23]. Conversely, in the lab, larvae are fed with micro-worms and artemia nauplii, and juveniles and adults are fed with granules that contain by-products of fish, cereals, vegetables, and crustaceans. Lab-raised fish eat very little decomposing food since they finish their meals in a few minutes. Such differences in feeding experience and odor exposure might explain the differential responses of Lab-Pachón and CF-Pachón. The repulsive behavior after alanine perfusion 
in the natural cave may suggest that CF-Pachón are exposed to an unpalatable organic matter that gives off alanine. We do not know the amino acids compositions of different organic materials found in this cave. It is therefore difficult to determine which one might be the source of this repulsion. Overall, the sometimes-contrasting responses that we observed in the lab and in the wild reinforce the interest and necessity to assess behavioral repertoires in the natural environment to discuss ecological or evolutionary relevance.

\subsection{Not All Cavefish Respond the Same}

Our field observations in five different caves lead to one main conclusion: Cavefish from different caves showed very distinctive responses when presented with exogenous odor cues at low concentrations (Table 1). For those that did respond (CF-Pachón, CF-SubT), we can propose that they have augmented olfactory skills when compared to their surface conspecifics, which confirms our previous studies $[14,20]$. For those which did not respond as well (CF-Sabinos) or did not respond at all (CF-Chica, CF-Tinaja), several questions arise. Is the lack of response due to local environment, previous experience and/or fish condition and nutritional status? Or is it due to experimental conditions?

(1) Let us start with the latter-perhaps less biologically interesting-hypothesis. We cannot rule out that CF-Chica did not respond because the cave water used (although filtered) was so loaded with debris and dissolved organic materials and endogenous olfactory cues that their olfactory system was saturated and could not detect the low concentrations of amino acids or even chondroitin perfused (see Figure 4E). The same holds true in the Tinaja cave, where carbon content in the mud is high (32\%, to be compared to 9.2\% in the main pool of Pachón) [24], and, maybe to a lesser extent, in the Sabinos cave (see description in Results). A way to test directly this hypothesis would be to transport "clean" tap water into the caves to perform the experiments. However, we feel that the interest of such experiments would be rather limited, as we anticipate that they would be flawed with other problems and quite demanding in terms of logistics. Nevertheless, future studies examining the physicochemical parameters and the exact nature of compounds present in the water will be important to answer these questions.

(2) The environment-dependent and experience-dependent hypothesis is more scientifically exciting to discuss adaptation to the environment. It implies that the expression of olfactory skills depends on local environment, such as the configuration and ecological parameters of the cave and previous olfactory experiences of the fish related to these local environmental parameters, including possible interactions with other sensory systems and fish nutritional status and motivation to find food.

Indirect support for this hypothesis comes from our finding that CF-Pachón and CF-SubT, which do not belong to the same geographical group of caves and correspond to independently evolved cavefish populations $[5,11,48]$, both show significantly augmented olfactory skills. Thus, this trait most probably corresponds to an evolutionary convergence. This result further suggests that the evolution of food-related (and maybe pheromonal, not addressed here) odor sensing is of paramount importance for cavefish life, adaptation, and survival in the dark. On the other hand, population genetic studies have shown that El Abra cave populations (Pachón, Sabinos, Tinaja, Chica) are genetically close and share many polymorphisms $[25,48,49]$, suggesting that CF-Sab or CF-Tinaja probably carry all or part of the mutations in "olfaction genes" that genetically determine CF-Pachón olfactory skills. However, they do not necessarily express these skills as a consequence of plasticity, with expression depending on the environmental conditions at the precise time and location where we measured olfactory responses. The most striking pieces of evidences in favor of this possibility are discussed below.

CF-Pachón were the smallest among all fish assayed in this study, in agreement with previous measurements [24]. They live in a low-carbon cave (9.2\% in the mud), with limited influx of organic matter from the surface. There, foraging must be challenging and strongly olfactory-driven. Conversely, CF-Chica were the largest and biggest among the cavefish that we tested and measured. The very large bat colonies in this "dirty" cave probably render foraging easy, together with fast 
growth and a long lifespan. CF-Tinaja are also large and old [24] and live in a carbon-rich (31.9\%) environment [23]. Strikingly, and in line with these very different trophic environmental conditions, CF-Pachón and CF-Chica (and CF-Tinaja, according to preliminary results) were at the two ends of the spectrum of olfactory responses that we observed.

Vibration attraction behavior (VAB) is thought to help cavefish locate food droppings or vibrating objects at the surface of the water $[18,50]$. VAB is mediated by neuromasts, and therefore corresponds to a mechanosensory modality. In the field, VAB responses are variable in different caves and even in different ponds of a single cave [51]. In cave ponds where insects are numerous or where percolating water drops are abundant, food search is probably strongly guided by VAB, and less so by the chemosensory olfactory modality. Our results fit well with this idea. Indeed, in the Tinaja cave, where it literally "rains," or in the Sabinos cave, where we personally witnessed abundant water drops over pool 1 to which the CF-Sab were systematically attracted (SR and A. Alié, pers.obs.), the olfactory sense and skills may not be solicited as much as in the Pachón or Subterráneo caves, where vibrating objects are much rarer and food must be found by the nose. Thus, we propose the possibility of a balanced use of different sensory modalities and expression of sensory skills depending on local conditions. Of note, fish may preferentially use one or the other (or both) sense across their lifespan to find a proper diet and to adapt to different locations where they can swim in a cave, or even across seasonal fluctuations of local ecological conditions.

Finally, cavefish genetics and the hybridization with introgressed surface fish is also a parameter to consider. Whereas Pachón, Sabinos, and Tinaja cavefish are fully troglomorphic (see Figure 3), CF-SubT and CF-Chica correspond to "hybrid-like" populations and carry surface fish alleles (see [14]). Interestingly, the entrance of surface individuals into these two caves does not proceed by the same end: In Subterráneo, surface fish are washed inside the cave from the surface by flooding, whereas, in Chica, surface fish enter the cave by a bottom resurgence. This probably explains why, in Chica, the pool 2 (deeper) fish, which carry more surface alleles, are smaller than the pool 1 (upper) fish, which are less hybridized and more troglomorphic and cave-adapted. In any case, we cannot exclude that the important gene flow from the surface that exists in Chica $[25,48]$ might counteract the effects of cave alleles, favoring the evolution of augmented olfactory skills. Hence, the absence of olfactory responses in CF-Chica might correspond to truly modest olfactory capacities. Deciphering the effects of genetics (presence of surface alleles) from the effects of environmental parameters on plasticity (food-rich cave) or from experimental conditions (odor-saturated water during test) is impossible at this stage. However, it must be noted that the CF-SubT, which also carry surface alleles, responded much better than CF-Chica to the odors we presented: They strongly responded to both alanine and chondroitin. Therefore, in this later case, the effects of hybridization and introgression of surface alleles appears very limited on the behavioral phenotype.

\section{Conclusions}

Almost 80 years have passed since the first descriptions of ecological conditions in which Chica cavefish live by Charles Breder [52]. Since then, fieldwork in Astyanax caves has continuously brought novel information on caves topography, population genetics, and cavefish biology in general. Recently, important examples of insights on the evolution of cavefish behaviors or the evolution of their immune system started from field observations [53,54]. Here, by bringing the "behavior room" into the field, we highlighted the diversity and complexity of the mechanisms that underlie cave adaptation and documented the diversity of cave environments inhabited by a single species of cavefish, A. mexicanus. Our data confirm the classical proposal of sensory compensations to the absence of vision in the dark. Indeed, overall cavefish showed lower (i.e., better) olfactory detection thresholds than surface fish. However, the picture appears more complicated than simply "cavefish smell better." The next challenge will be to disentangle the effects of genetics, plasticity, environment, and their interactions in the evolution of cavefish olfactory system. Then, of course, more fieldwork will be needed to refine 
behavioral observations and to further describe the diverse natural ecological conditions in which cavefish live.

Supplementary Materials: The following are available online at http://www.mdpi.com/1424-2818/12/10/0395/s1, Figure S1: Rationale for olfactory scoring. Figure S2: Place preference problem in the Sabinos cave. Video S1: Behavioral response of Pachón cavefish to chondroitin in laboratory settings. Video S2: Behavioral response of Pachón cavefish to chondroitin in natural settings.

Author Contributions: Conceptualization, M.B. and S.R.; methodology, M.B., J.F. and S.R.; validation, M.B. and S.R.; formal analysis, M.B. and S.R.; investigation, all authors; data curation, M.B.; writing-original draft preparation, S.R. and M.B.; writing-review and editing, S.R.; visualization, M.B. and S.R.; supervision, S.R.; project administration, S.R.; funding acquisition, S.R. All authors have read and agreed to the published version of the manuscript.

Funding: This research was funded by CNRS, ANR (Agence Nationale pour la Recherche) grant [BLINDTEST], Fondation pour la Recherche Médicale Equipe FRM grant [DEQ20150331745] to SR, and a French-Mexican Ecos-Nord Exchange Program grant [M15A03] to SR and Patricia-Ornelas Garcia (UNAM, Mexico). The APC was funded by CNRS.

Acknowledgments: We thank Laurent Legendre, Luis Espinasa, Didier Casane, Carole Hyacinthe, Victor Simon, Eric Queinnec, Karen Pottin, Stéphane Rode, Joel Attia and all other past and present members of the Rétaux's lab for their invaluable help and team spirit during fieldwork over the years. We are grateful to Krystel Saroul for taking care of our lab Astyanax colony.

Conflicts of Interest: The authors declare no conflict of interest.

\section{References}

1. Culver, D.C.; Pipan, T. The Biology of Caves and Other Subterranean Habitats, 2nd ed.; Biology of Habitats Series; Oxford University Press: Oxford, UK, 2019; ISBN 978-0-19-882077-2.

2. Rétaux, S.; Casane, D. Evolution of eye development in the darkness of caves: Adaptation, drift, or both? EvoDevo 2013, 4, 26. [CrossRef] [PubMed]

3. Romero, A. Cave Biology: Life in Darkness. Integr. Comp. Biol. 2010, 50, 689-691. [CrossRef]

4. Simon, V.; Hyacinthe, C.; Rétaux, S. Breeding behavior in the blind Mexican cavefish and its river-dwelling conspecific. PLoS ONE 2019, 14, e0212591. [CrossRef]

5. Mitchell, R.; Russell, W.; Elliott, W. Mexican eyeless characin fishes, genus Astyanax: Environment, distribution, and evolution. Spec. Publ. Mus. Texas Tech. Univ. 1977, 12, 1-89.

6. Elliott, W.R. Cave Biodiversity and Ecology of the Sierra de El Abra Region. In Biology and Evolution of the Mexican Cavefish; Elsevier: Amsterdam, The Netherlands, 2016; pp. 59-76. ISBN 978-0-12-802148-4.

7. Casane, D.; Rétaux, S. Evolutionary Genetics of the Cavefish Astyanax mexicanus. Adv. Genet. 2016, 95, 117-159. [CrossRef]

8. Jeffery, W.R. Chapter 8 Evolution and Development in the Cavefish Astyanax. In Current Topics in Developmental Biology; Elsevier: Amsterdam, The Netherlands, 2009; Volume 86, pp. 191-221. ISBN 978-0-12-374455-5.

9. Keene, A.; Yoshizawa, M.; Mcgaugh, S.E. Biology and Evolution of the Mexican Cavefish; Elsevier Science: Amsterdam, The Netherlands, 2015; ISBN 978-0-12-802148-4.

10. Espinasa, L.; Legendre, L.; Fumey, J.; Blin, M.; Rétaux, S.; Espinasa, M. A new cave locality for Astyanax cavefish in Sierra de El Abra, Mexico. Subterr. Biol. 2018, 26, 39-53. [CrossRef]

11. Elliott, W.R. The Astyanax Caves of Mexico Cavefishes of Tamaulipas, San Luis Potosi, and Guerrero; Association for Mexican Cave Studies: Austin, TX, USA, 2018.

12. Şadoğlu, P. A mendelian gene for albinism in natural cavefish. Experientia 1957, 13, 394. [CrossRef]

13. Borowsky, R. Restoring sight in blind cavefish. Curr. Biol. CB 2008, 18, R23-R24. [CrossRef]

14. Bibliowicz, J.; Alié, A.; Espinasa, L.; Yoshizawa, M.; Blin, M.; Hinaux, H.; Legendre, L.; Père, S.; Rétaux, S. Differences in chemosensory response between eyed and eyeless Astyanax mexicanus of the Rio Subterráneo cave. EvoDevo 2013, 4, 25. [CrossRef]

15. Fumey, J.; Hinaux, H.; Noirot, C.; Thermes, C.; Rétaux, S.; Casane, D. Evidence for late Pleistocene origin of Astyanax mexicanus cavefish. BMC Evol. Biol. 2018, 18, 43. [CrossRef]

16. Policarpo, M.; Fumey, J.; Lafargeas, P.; Naquin, D.; Thermes, C.; Naville, M.; Dechaud, C.; Volff, J.-N.; Cabau, C.; Klopp, C.; et al. Contrasting gene decay in subterranean vertebrates: Insights from cavefishes and fossorial mammals. Mol. Biol. Evol. 2020. [CrossRef] [PubMed] 
17. Yamamoto, Y.; Byerly, M.S.; Jackman, W.R.; Jeffery, W.R. Pleiotropic functions of embryonic sonic hedgehog expression link jaw and taste bud amplification with eye loss during cavefish evolution. Dev. Biol. 2009, 330, 200-211. [CrossRef] [PubMed]

18. Yoshizawa, M.; Gorički, Š.; Soares, D.; Jeffery, W.R. Evolution of a Behavioral Shift Mediated by Superficial Neuromasts Helps Cavefish Find Food in Darkness. Curr. Biol. 2010, 20, 1631-1636. [CrossRef] [PubMed]

19. Blin, M.; Tine, E.; Meister, L.; Elipot, Y.; Bibliowicz, J.; Espinasa, L.; Rétaux, S. Developmental evolution and developmental plasticity of the olfactory epithelium and olfactory skills in Mexican cavefish. Dev. Biol. 2018, 441, 242-251. [CrossRef]

20. Hinaux, H.; Devos, L.; Blin, M.; Elipot, Y.; Bibliowicz, J.; Alié, A.; Rétaux, S. Sensory evolution in blind cavefish is driven by early embryonic events during gastrulation and neurulation. Development 2016, 143, 4521-4532. [CrossRef]

21. Torres-Paz, J.; Hyacinthe, C.; Pierre, C.; Rétaux, S. Towards an integrated approach to understand Mexican cavefish evolution. Biol. Lett. 2018, 14. [CrossRef]

22. Parzefall, J. Field observation in epigean and cave populations of the Mexican characid Astyanax mexicanus (Pisces, Characidae). Mémoires de Biospéologie 1983, 10, 171-176.

23. Espinasa, L.; Bonaroti, N.; Wong, J.; Pottin, K.; Queinnec, E.; Rétaux, S. Contrasting feeding habits of post-larval and adult Astyanax cavefish. Subterr. Biol. 2017, 21, 1-17. [CrossRef]

24. Simon, V.; Elleboode, R.; Mahé, K.; Legendre, L.; Ornelas-Garcia, P.; Espinasa, L.; Rétaux, S. Comparing growth in surface and cave morphs of the species Astyanax mexicanus: Insights from scales. EvoDevo 2017, 8. [CrossRef]

25. Pierre, C.; Pradère, N.; Froc, C.; Ornelas-García, P.; Callebert, J.; Rétaux, S. A mutation in monoamine oxidase (MAO) affects the evolution of stress behavior in the blind cavefish Astyanax mexicanus. J. Exp. Biol. 2020. [CrossRef]

26. Strecker, U.; Bernatchez, L.; Wilkens, H. Genetic divergence between cave and surface populations of Astyanax in Mexico (Characidae, Teleostei). Mol. Ecol. 2003, 12, 699-710. [CrossRef] [PubMed]

27. Mathuru, A.S.; Kibat, C.; Cheong, W.F.; Shui, G.; Wenk, M.R.; Friedrich, R.W.; Jesuthasan, S. Chondroitin fragments are odorants that trigger fear behavior in fish. Curr. Biol. CB 2012, 22, 538-544. [CrossRef] [PubMed]

28. Pfeiffer, W. [On the heredity of the fear reaction in Astyanax (Characidae, pisces)]. Zeitschrift fur Vererbungslehre 1966, 98, 97-105. [PubMed]

29. Parzefall, J.; Fricke, D. Alarm reaction and schooling in population hybrids of Astyanax fasciatus (Pisces, Characidae). Memoires e Biospeologie 1991, 18, 29-32.

30. VideoLan. VLC Media Player. 2006. Available online: https://www.videolan.org/vlc/index.html (accessed on 1 February 2010).

31. Pérez-Escudero, A.; Vicente-Page, J.; Hinz, R.C.; Arganda, S.; de Polavieja, G.G. idTracker: Tracking individuals in a group by automatic identification of unmarked animals. Nat. Methods 2014, 11, 743-748. [CrossRef]

32. R Core Team. R: A Language and Environment for Statistical Computing; R Foundation for Statistical Computing: Vienna, Austria, 2017; Available online: https://www.R-project.org/ (accessed on 1 February 2018).

33. RStudio Team. RStudio: Integrated Development for R; RStudio, PBC: Boston, MA, USA; Springer: New York, NY, USA, 2020; Available online: http://www.rstudio.com/ (accessed on 1 February 2018).

34. Alboukadel Kassambara Rstatix: Pipe-Friendly Framework for Basic Statistical Tests. R Package Version 0.5.0. 2020. Available online: https://CRAN.R-project.org/package=rstatix (accessed on 1 February 2020).

35. Wickham, H. ggplot2: Elegant Graphics for Data Analysis; Springer: New York, NY, USA, 2016; ISBN 978-3-319-24277-4. Available online: https://ggplot2.tidyverse.org (accessed on 1 February 2020).

36. Wickham, H.; Averick, M.; Bryan, J.; Chang, W.; McGowan, L.D.A.; François, R.; Grolemund, G.; Hayes, A.; Henry, L.; Hester, J.; et al. Welcome to the tidyverse. J. Open Sour. Softw. 2019, 4, 1686. [CrossRef]

37. Elipot, Y.; Legendre, L.; Père, S.; Sohm, F.; Rétaux, S. Astyanax transgenesis and husbandry: How cavefish enters the laboratory. Zebrafish 2014, 11, 291-299. [CrossRef]

38. Hara, T.J. Olfaction and gustation in fish: An overview. Acta Physiol. Scand. 1994, 152, 207-217. [CrossRef]

39. Hara, T.J. Feeding behaviour in some teleosts is triggered by single amino acids primarily through olfaction. J. Fish. Biol. 2006, 68, 810-825. [CrossRef]

40. Friedrich, R.W.; Korsching, S.I. Combinatorial and chemotopic odorant coding in the zebrafish olfactory bulb visualized by optical imaging. Neuron 1997, 18, 737-752. [CrossRef]

41. Frisch, K. Die Bedeutung des Geruchsinnes im Leben der Fische. Süffert F Ed. Naturwissenschaften 1941, 321-333. [CrossRef] 
42. Døving, K.B.; Lastein, S. The alarm reaction in fishes-odorants, modulations of responses, neural pathways. Ann. N. Y. Acad. Sci. 2009, 1170, 413-423. [CrossRef] [PubMed]

43. Fricke, D. Reaction to Alarm Substance in Cave Populations of Astyanax fasciatus (Characidae, Pisces). Ethology 1987, 76, 305-308. [CrossRef]

44. Kowalko, J. Utilizing the blind cavefish Astyanax mexicanus to understand the genetic basis of behavioral evolution. J. Exp. Biol. 2020, 223. [CrossRef]

45. Vitebsky, A.; Reyes, R.; Sanderson, M.J.; Michel, W.C.; Whitlock, K.E. Isolation and characterization of the laure olfactory behavioral mutant in the zebrafish, Danio rerio. Dev. Dyn. 2005, 234, 229-242. [CrossRef]

46. Braubach, O.R.; Wood, H.-D.; Gadbois, S.; Fine, A.; Croll, R.P. Olfactory conditioning in the zebrafish (Danio rerio). Behav. Brain Res. 2009, 198, 190-198. [CrossRef]

47. Valentinčič, T.; Wegert, S.; Caprio, J. Learned olfactory discrimination versus innate taste responses to amino acids in channel catfish (Ictalurus punctatus). Physiol. Behav. 1994, 55, 865-873. [CrossRef]

48. Bradic, M.; Beerli, P.; García-de León, F.J.; Esquivel-Bobadilla, S.; Borowsky, R.L. Gene flow and population structure in the Mexican blind cavefish complex (Astyanax mexicanus). BMC Evol. Biol. 2012, 12, 9. [CrossRef]

49. Avise, J.C.; Selander, R.K. Evolutionary genetics of cave-dwelling fishes of the genus astyanax. Evol. Int. J. Org. Evol. 1972, 26, 1-19. [CrossRef]

50. Yoshizawa, M.; Jeffery, W.R. Evolutionary tuning of an adaptive behavior requires enhancement of the neuromast sensory system. Commun. Integr. Biol. 2011, 4, 89-91. [CrossRef]

51. Espinasa, L.; Heintz, C.; Rétaux, S.; Yoshisawa, M.; Agnès, F.; Ornelas-Garcia, P.; Balogh-Robinson, R. Vibration Attraction Response (VAB) is a plastic trait in Blind Mexican tetra (Astyanax mexicanus), variable within subpopulations inhabiting the same cave. J. Fish. Biol. 2020. [CrossRef]

52. Breder, C.M. Descriptive ecology of La Cueva Chica, with especial reference to the blind fish, Anoptichthys. Zoologica 1942, 27, 7-15.

53. Hyacinthe, C.; Attia, J.; Rétaux, S. Evolution of acoustic communication in blind cavefish. Nat. Commun. 2019, 10, 4231. [CrossRef]

54. Peuß, R.; Box, A.C.; Chen, S.; Wang, Y.; Tsuchiya, D.; Persons, J.L.; Kenzior, A.; Maldonado, E.; Krishnan, J.; Scharsack, J.P.; et al. Adaptation to low parasite abundance affects immune investment and immunopathological responses of cavefish. Nat. Ecol. Evol. 2020. [CrossRef]

(C) 2020 by the authors. Licensee MDPI, Basel, Switzerland. This article is an open access article distributed under the terms and conditions of the Creative Commons Attribution (CC BY) license (http://creativecommons.org/licenses/by/4.0/). 